\title{
A Prospective Study on Effectiveness of Sitagliptin with Metformin in Type 2 Diabetic Patients in a Tertiary Care Hospital
}

\author{
Preeti Singh ${ }^{*}$, VK Singh ${ }^{2}$, TJ Hemnani ${ }^{3}$, Prithpal S Matreja ${ }^{4}$ \\ ${ }^{1 *}$ Assistant Professor, 4Professor, Department of Pharmacology, ${ }^{2}$ Professor, Department of Medicine, \\ Teerthanker Mahaveer Medical College \& Research Centre, TMU, Moradabad, Uttar Pradesh, India. \\ 3Principal \& Professor Pharmacology, GS Medical College \& Hospital, Pilkhuwa, Hapur, Uttar Pradesh, India.
}

\begin{abstract}
Background: Type $2 \mathrm{DM}$ is one of the most important non communicable disease of world, mainly in developing countries and constitutes a substantial burden for both the patients and health care system. Its incidence is increasing day by day as every $5^{\text {th }}$ patient visiting a consulting physician is diabetic. Treatment with Sitagliptin achieved adequate reductions in the levels of $\mathrm{HbA} 1 \mathrm{c}$ and significant increases in the rate of accomplishment of glycemic control. The use of Sitagliptin was shown to be safe and improved the Diabetes Symptomatic scores.
\end{abstract}

Methods: This prospective study was carried out on Type 2 Diabetic patients attending the Diabetic clinic at Teerthanker Mahaveer Medical College \& Research Center, Moradabad. Only those patients were included who were not adequately controlled with a stable dose of metformin monotherapy. A total of 109 patients were enrolled in the study taking sitagliptin plus Metformin. The patients received therapy metformin $500 \mathrm{mg}$ three times daily and sitagliptin $100 \mathrm{mg}$ once daily.

Results: Out of the 109 patients enrolled in the study, only 79 patients completed the study. Out of 79 patients, 47 were males and 32 females. There was a significant reduction in the mean percentage of PPBS. There was a significant reduction $(p<0.05)$ of FPG as compared to baseline. The FPG was reduced in Group B from $189.15 \pm 32.37$ (mg/dl) to $03.66 \pm 11.266$ at 24 weeks. The (PPBS) was reduced from

\section{INTRODUCTION}

DPP-4 inhibitor Sitagliptin has beneficial effects not only for glycemic control, but also for improving blood pressure, lipid profiles, and quality of life regarding sleep quality and diabetes symptoms in addition to being safe with a high rate of adherence to treatment. Generally, Sitagliptin was well tolerated drug. Compared to treatment with glimepiride, treatment with Sitagliptin was associated with a lower risk of hypoglycemia and with weight loss versus weight gain. DPP IV prolongs the duration of active glucagon-like peptide 1 (GLP-1) by inhibiting DPPIV peptidase, an enzyme which cleaves the active form of the peptide. This action results in an improvement of insulin secretion as a physiological response to feeding. The mechanism of DPPIV inhibitors is complementary to that of metformin which improves insulin sensitivity and reduces hepatic glucose production, making this combination very useful for achieving adequate glycemic control. ${ }^{1}$ Systolic and diastolic blood pressure decreased after 3 -months of treatment with Sitagliptin.

The efficacies of these agents have been established in several randomized controlled trials. These agents are well tolerated,
$300.17 \pm 62.882(\mathrm{mg} / \mathrm{dl})$ to $134.21 \pm 14.110(\mathrm{mg} / \mathrm{dl})$ at 24 weeks. There was a significant reduction $(p<0.05)$ of $\mathrm{Hb} 1 \mathrm{Ac}, \mathrm{TC}, \mathrm{TAG}$, LDL, VLDL, UA, SGOT as compared to baseline but no significant rise in HDL was seen as compared to baseline.

Conclusion: Significant improvement in fasting blood sugar (FBS), Postprandial blood sugar (PPBS), Lipid profile and Glycosylated hemoglobin as compared to baseline.

Keywords: Metformin, sitagliptin, type 2 Diabetes Mellitus, Metabolic Syndrome.

\section{${ }^{*}$ Correspondence to:}

Dr. Preeti Singh, Assistant Professor, Department of Pharmacology, Teerthanker Mahaveer Medical College \& Research Centre, TMU.

Email: drpreetisinghtmu@gmail.com

\section{Article History:}

Received: 03-05-2016, Revised: 12-05-2016, Accepted: 30-05-2016

\begin{tabular}{|l|c|}
\hline \multicolumn{2}{|c|}{ Access this article online } \\
\hline Website: & Quick Response code \\
www.jimrp.com & \\
\hline DOl: & \\
10.21276/jijm.2016.2.3.054 & \\
\hline
\end{tabular}

carry a low risk of hypoglycemia, and are weight-neutral. ${ }^{2}$ Several published studies propose that DPP-4 inhibitors may have a protective cardiovascular effect. Suggested mechanisms include improved heart function and coronary perfusion, reduced blood pressure, and favorable effects on the lipid profile. ${ }^{3,4}$ Further data are needed to determine whether DPP-4 inhibition improves cardiovascular outcomes in type-2 diabetes. Expected reductions in $\mathrm{HbA}_{1 \mathrm{c}}$ values with the use of DPP-4 inhibitors range from 0.5 to $0.8 \%$.

Although clinical trials of DPP-4 inhibitors suggested an increased risk of upper respiratory tract infections, urinary tract infections, and nasopharnyngitis, a meta-analysis published in 2012 did not find any increased risk compared with other treatments. ${ }^{5}$ Dosage adjustments are required for both sitagliptin and saxagliptin in patients with renal disease, whereas no dosage alterations are necessary for linagliptin, which might be preferred in patients with significant renal disease. ${ }^{6}$ DPP-4 inhibitor therapy should be discontinued in patients presenting with acute pancreatitis, and patients should not be rechallenged. 
DPP-4 inhibitors provide a safe and effective option for patients with type-2 diabetes and may be preferred in elderly individuals, given their weight-neutrality, low propensity for hypoglycemia, and safety in renal impairment. ${ }^{4}$

\section{METHODOLOGY}

This study was carried out at Teerthanker Mahaveer Medical College \& Research Center, Moradabad for the consecutive newly diagnosed diabetic patients $>40(40-70)$ years of age attending the Diabetic clinic and diagnosed with Type 2 diabetes using the standard diagnostic criteria in effect at the inception of the study. In this prospective study, patients were included with a history of T2DM not adequately controlled with a stable dose of metformin monotherapy

A total of 109 patients were enrolled in the study taking sitagliptin plus Metformin. The patients received therapy metformin $500 \mathrm{mg}$ three times daily and sitagliptin $100 \mathrm{mg}$ once daily.

\section{Diagnosis of Diabetes}

Diagnosis of the patients suffering from type 2 diabetes mellitus was made on the basis of clinical assessment and diagnostic tests as per the ADA Guidelines. According to ADA, the fasting plasma glucose (FPG), 2 hours post meal blood glucose, random blood sugar is reliable and convenient test to identity diabetes in asymptomatic subjects.

\section{Parameters measured}

The patients were advised follow up at 12 weeks and 24 weeks. At each visit patients were evaluated for glycemic parameters and biochemical parameters. The glycemic parameters included analysis of Fasting plasma glucose, post prandial plasma glucose and glycosylated hemoglobin. The biochemical analysis included lipid profile - total cholesterol, serum triglycerides level, lowdensity lipoprotein-cholesterol (LDL-C), high-density lipoproteinprotein and very low density lipoprotein estimation. The safety analysis was performed by measuring serum creatinine, serum uric acid level, SGOT and SGPT level at 12 weeks and 24 weeks. The follow up of those patients continued for six months and blood samples for various estimation withdrawn at base line 3 month and 6 months of the study.

\section{Statistical Analysis:}

Data was analyzed using SPSS version 17.

\section{RESULTS}

Out of the 109 patients enrolled in our study, only 79 patients completed the study (Table 1). Out of 79 patients, 47 males and 32 females. Mean age of group is $54.34 \pm 10.52$ years, Mean weight was $70.83 \pm 9.82$ Kilogram $(\mathrm{Kg})$, Mean Fasting blood sugar (FBS) was found to be $189.15 \pm 32.37$ (mg/dl), Mean Postprandial blood sugar (PPBS) was $300.17 \pm 62.88(\mathrm{mg} / \mathrm{dl})$, Mean Total cholesterol (TC) was $210.40 \pm 39.29(\mathrm{mg} / \mathrm{dl})$, Mean Triglyceride (TG) is $180.47 \pm 57.60$ (mg/dl), Mean Low Density Protein (LDL) is $138.14 \pm 44.63(\mathrm{mg} / \mathrm{dl})$. Mean Very Low Density Protein (VLDL) was $31.42 \pm 12.88(\mathrm{mg} / \mathrm{dl})$, mean $\mathrm{HDL}$ is $48.94 \pm 12.88(\mathrm{mg} / \mathrm{dl})$ mean UA was $4.46 \pm 1.22(\mathrm{mg} / \mathrm{dl})$, mean $\mathrm{SC}$ is $0.88 \pm 0.24$ $(\mathrm{mg} / \mathrm{dl})$, mean SGOT is $39.60 \pm 6.92(\mathrm{IU} / \mathrm{L})$ and mean SGPT is $44.43 \pm 9.20$ (IU/L). (Table 1)

Table 1. Metabolic parameters at baseline, 12 and 24 weeks.

\begin{tabular}{llccc}
\hline S.N & & Baseline & 12 Weeks & 24 Weeks \\
\cline { 3 - 5 } & & & (Mean $\mathbf{n}$ SD) & \\
$\mathbf{n}$ & Age (years) & $54.34 \pm 10.52$ years & & \\
$\mathbf{2}$ & Weight(Kilogram) & $70.83 \pm 9.82$ & & \\
$\mathbf{3}$ & Fasting Blood Sugar (FBS (mg/dl) & $189.15 \pm 32.37$ & $128.40 \pm 19.893^{*}$ & $103.66 \pm 11.266^{*}$ \\
$\mathbf{4}$ & Postprandial Blood Sugar (PPBS) (mg/dl) & $300.17 \pm 62.88$ & $168.74 \pm 26.469^{*}$ & $134.21 \pm 14.110^{*}$ \\
$\mathbf{5}$ & Glycosylated Hemoglobin (Hb1Ac) (mmol/mol) & $9.39 \pm 0.82$ & $134.21 \pm 14.110^{*}$ & $6.838 \pm .3566^{*}$ \\
$\mathbf{6}$ & Total Cholesterol (TC) (mg/dl) & $210.40 \pm 39.29$ & $183.19 \pm 37.087^{*}$ & $146.09 \pm 27.786^{*}$ \\
$\mathbf{7}$ & Serum Trigylcerides (TG) (mg/dl) & $180.47 \pm 57.60$ & $150.77 \pm 48.218^{*}$ & $119.02 \pm 29.559^{*}$ \\
$\mathbf{8}$ & Low Density Lipoprotein (LDL) (mg/dl) & $138.14 \pm 44.63$ & $107.37 \pm 28.950^{*}$ & $74.26 \pm 21.117^{*}$ \\
$\mathbf{9}$ & Very Low Density Lipoproteins (VLDL) (mg/dl) & $31.42 \pm 12.88$ & $26.53 \pm 11.105^{*}$ & $19.93 \pm 10.180^{*}$ \\
$\mathbf{1 0}$ & High Density Lipoprotein (HDL) (mg/dl) & $48.94 \pm 12.88$ & $50.26 \pm 2.428^{*}$ & $50.26 \pm 12.428$ \\
$\mathbf{1 1}$ & Serum Uric Acid (UA) (mg/dl) & $4.46 \pm 1.22$ & $4.24 \pm 0933^{*}$ & $4.271 \pm 1.0423$ \\
$\mathbf{1 2}$ & Serum Creatinine (SC) (mg/dl) & $0.88 \pm 0.24$ & $0.873 \pm .2094$ & $0.8594 \pm .17659$ \\
$\mathbf{1 3}$ & Aspartate Aminotransferase (SGOT) (IU/L) & $39.60 \pm 6.92$ & $38.06 \pm 5.708^{*}$ & $36.53 \pm 5.729^{*}$ \\
$\mathbf{1 4}$ & Alanine transaminase (SGPT) (IU/L) & $39.60 \pm 6.92$ & $41.02 \pm 7.128^{*}$ & $38.45 \pm 6.430^{*}$ \\
\hline
\end{tabular}

$p>0.05$ as compared to other group using student unpaired ' $t$ ' test

${ }^{*} p<0.05$ within group by using student paired ' $t$ ' test

${ }^{\#} p<0.05$ as compared to other group using student unpaired ' $\mathrm{t}$ ' test

\section{DISCUSSION}

T2DM is a chronic metabolic disorder \& has high prevalence in India. T2DM is characterized by a long asymptomatic period of hyperglycemia and many individuals with T2DM have complications even at the time of diagnosis. Hyperglycemia is associated with number of microvascular and macrovascular complications, which are significant cause of morbidity \& mortality among diabetes subjects.
This study was undertaken to assess the differential effect on glucose and lipid parameters of glimepiride plus metformin in patients suffering from type 2 diabetes and metabolic syndrome. Our study found that there was significant improvement in fasting plasma glucose, Postprandial blood glucose, cholesterol profile, and $\mathrm{HbA} 1 \mathrm{c}$ at 12 weeks and 24 weeks as compared to baseline. Triglycerides (TAG), (LDL) in metformin and sitagliptin group at 24 
weeks. There was also significant percentage change in total Cholesterol ( $T$ C) and High Density Lipoprotein (HDL) in metformin and sitagliptin group at 12 weeks. A better change was observed in metformin and sitagliptin group at 24 weeks. Both the therapies were well tolerated by the patients. Another retrospective study done by Kumar $S$ et.al., to investigate the effects of adding sitagliptin or glimepiride on treatment success, safety and patient satisfaction in 100 patients with type 2 diabetes showed that the $\mathrm{HbA} 1 \mathrm{c}$ level after the treatment of 12-24 weeks was not significant as compared to each other or from control.

Comparison between the groups showed significant good results in PPBS in group with metformin and sitagliptin at the completion of the study. This is similar result with our study as significant percentage reduction in PPBS at 24 weeks in metformin and sitagliptin.

In 2007 a study done by K. Hermansen etal. to analyse the efficacy and safety of a 24-week treatment with sitagliptin in patients suffering from type 2 diabetes whose blood glucose levels was not controlled on glimepiride alone or in combination with metformin.

After 24 weeks of treatment, sitagliptin decreased $\mathrm{HbA} 1 \mathrm{c}$ by $0.74 \%$ relative to placebo. In the patients taking glimepiride plus metformin, sitagliptin decreases $\mathrm{HbA}_{1 \mathrm{c}}$ by $0.89 \%$ relative to placebo, compared with a decrease of $0.57 \%$ in the subset of patients taking glimepiride only. Thus this study shows that Sitagliptin significantly improved glucose control and $\beta$-cell function in patients suffering from type 2 diabetes whose blood glucose levels were not controlled with glimepiride or glimepiride plus metformin therapy. A study done by Giuseppe Derosa to evaluate the effects of sitagliptin + metformin compared to metformin in T2DM diabetic patients on glycemic control, insulin resistance, and insulin secretion and they found that Other than improving glycemic control, sitagliptin + metformin also improved $\beta$-cell function better than metformin alone. In our study there was also significant improvement in glycemic control but we have not included $\beta$-cell function parameter in our study. lipid profile was also included in our study and there was significant improvement.

There are certain limitations in our study, firstly, our study is an open-label study, a double blind study would have been appropriate, but due to lack of funds and sponsorship, the patients had to routinely purchase the medication. Hence we stuck to an open label study only.

Secondly, the sample size is small, a large sample size could have given different results. The time period for enrollment of patients was only one year and patients had to be followed up for a duration of 6 months so it would have not been feasible if the sample size was large.

Thirdly, the duration of study was only 6 months, a larger duration study could have given different results. Increasing the time to follow up was not possible as the duration for enrollment of patients was only one year with 6 months follow up, if any aspect of these two would have been compromised, the study could not be completed.

\section{CONCLUSION}

Based on the findings of the present study it's possible to conclude: it had significant improvement in fasting blood sugar (FBS), postprandial blood sugar (PPBS), Lipid profile, and Glycosylated hemoglobin as compared to baseline. At 12 weeks' Significant improvement in following parameters: Fasting blood sugar (FBS), Glycosylated Hemoglobin ( $\mathrm{HbA1c})$, Total Cholesterol (TC), Very Low Density Lipoprotein (VLDL).

\section{REFERENCES}

1. Richter B, Bandeira-Echtler $E$, Bergerhoff $K$, Lerch $C L$ : Dipeptidyl peptidase-4 (DPP-4) inhibitors for type 2 diabetes mellitus. Cochrane Database Syst Rev 2008, (2):CD006739.

2. Mannucci $E$, Ognibene A, Cremasco F: Effect of metformin on glucagon-like peptide 1 (GLP-1) and leptin levels in obese nondiabetic subjects. Diabetes Care 2001, 24:489-494.

3. Gerrald KR, Van Scoyoc E, Wines RC, et al. Saxagliptin and sitagliptin in adult patients with type 2 diabetes: A systematic review and meta-analysis. Diabetes Obes Metab. 2012;14(6):1481-1492. [PubMed]

4. Dicker D. DPP-4 inhibitors: Impact on glycemic control and cardiovascular risk factors. Diabetes Care.2011;34(Suppl 2):S276-S278. [PMC free article] [PubMed]

5. Karagiannis T, Paschos $P$, Paletas $K$, et al. Dipeptidyl peptidase-4 inhibitors for treatment of type 2 diabetes mellitus in the clinical setting: Systematic review and meta-analysis. BMJ. 2012;344:e1369.[PubMed]

6. Toth PP. Linagliptin: A new DPP-4 inhibitor for the treatment of type 2 diabetes mellitus. Postgrad Med.2011;123:46-53. [PubMed]

7. Inzucchi SE, Bergenstal RM, Buse JB, et al. Management of hyperglycemia in type 2 diabetes: A patient-centered approach: Position Statement of the American Diabetes Association (ADA) and the European Association for the Study of Diabetes (EASD) Diabetes Care. 2012;35:1364-1379. [PMC free article][PubMed]

\section{Source of Support: Nil.}

\section{Conflict of Interest: None Declared.}

Copyright: (c) the author(s) and publisher. IJMRP is an official publication of Ibn Sina Academy of Medieval Medicine \& Sciences, registered in 2001 under Indian Trusts Act, 1882.

This is an open access article distributed under the terms of the Creative Commons Attribution Non-commercial License, which permits unrestricted non-commercial use, distribution, and reproduction in any medium, provided the original work is properly cited.

Cite this article as: Preeti Singh, VK Singh, TJ Hemnani, Prithpal S Matreja. A Prospective Study on Effectiveness of Sitagliptin with Metformin in Type 2 Diabetic Patients in a Tertiary Care Hospital. Int J Med Res Prof. 2016; 2(3):246-48. 